\title{
A propos de Paso Doble, présentation et extraits de la vidéo-performance
}

Paul Ardenne et Miquel Barceló

\section{(2) OpenEdition}

12 Journals

Édition électronique

URL : https://journals.openedition.org/actesbranly/423

DOI : $10.4000 /$ actesbranly.423

ISSN : 2105-2735

Éditeur

Musée du quai Branly Jacques Chirac

Référence électronique

Paul Ardenne et Miquel Barceló, « A propos de Paso Doble, présentation et extraits de la vidéoperformance », Les actes de colloques du musée du quai Branly Jacques Chirac [En ligne], 2 | 2009, mis en ligne le 27 novembre 2009, consulté le 21 septembre 2021. URL : http://journals.openedition.org/ actesbranly/423; DOI : https://doi.org/10.4000/actesbranly.423

Ce document a été généré automatiquement le 21 septembre 2021.

(c) Tous droits réservés 


\title{
A propos de Paso Doble, présentation et extraits de la vidéo-performance
}

\author{
Paul Ardenne et Miquel Barceló
}

1 Paul Ardenne : Le travail de Miquel Barceló réalisé dans le cadre du festival d'Avignon, au cloître des Célestins, avec l'artiste Josef Nadj. Paso Doble, est le nom d'une danse espagnole. Dans un premier temps seront présentés des extraits de cette réalisation à quatre mains, une performance un peu particulière, qui a été rejouée à Avignon et redonnée à plusieurs occasions. Elle dure environ 40 minutes. Dans un second temps, il s'agira d'établir des connections, si c'est possible, entre l'art contemporain, la performance en art contemporain et les rituels.

2 Miquel Barceló, (en commentant la vidéo de la performance) : On est dans un contexte devant un mur d'argile et un sol d'argile. Ce sont des matières molles. Le mur est légèrement peint de blanc. Au début il $\mathrm{y}$ a un silence et on commence à voir le mur bouger par derrière. On est derrière le mur. C'est une représentation de ma manière de travailler l'argile. Finalement, c'est une espèce de forme de peinture. J'ai travaillé un grand mur d'argile qu'ensuite j'ai fait cuire. L'œuvre se détruit à chaque fois, l'œuvre est éphémère et j'ai travaillé avec coups de poings par derrière.

Paul Ardenne : Josef Nadj fait la même chose, il est éloigné de quelques mètres : est-ce que vous travaillez de manière synchrone? Vous vous êtes mis d'accord avant de commencer où est-ce qu'il t'imite ou est-ce toi qui l'imite?

4 Miquel Barceló On s'est mis un peu d'accord. Josef Nadj est chorégraphe, on pourrait dire qu'il est comme un : métronome. Il répète les gestes au millimètre près; ce qui me permet d'improviser à chaque fois. C'est ce qui rend supportable le fait de répéter la performance: je l'ai fais à Avignon avec la condition de ne le faire qu'une semaine et ensuite plus jamais. Alors puisqu'on l'a répété plein de fois à New York, à Londres, Athènes et Venise bientôt mon plaisir c'est de renouveler à chaque fois, en créant de nouvelles images.

5 Paul Ardenne : D'où vient l'idée de faire ce travail ? 
6 Miquel Barceló : En 2000, j'étais à Naples à Vietri sul Mare en train de travailler sur une œuvre énorme : le mur de terre cuite que j'ai faite pour la cathédrale de Palma à Majorque. C'est quand ça commence à sécher, quand ça commence à se fissurer que j'ai entrepris de faire la cuisson. C'était comme un puzzle sur le mur, n'est-ce pas, les craquelures. Et la technique de travail, c'était les coups de poings par devant et par derrière. Et puis je faisais les têtes de poisson qui était un peu comme ça [il indique un passage du film à l'écran]. Alors ça devient un poisson, un cœur qui bat, une gueule qui s'ouvre. Chaque nouvel accident devenait une image, souvent une image du monde organique. Ensuite j'ai montré à Nadj comment j'avais procédé et lui m’a invité à faire quelque chose à Avignon. Mais moi je pensais de rester dehors, je ne pensais pas me mettre en scène moi-même. Mon idée c'était, comment dire... j'étais arrivé à ça en faisant des grandes céramiques dans lesquels je rentrais dans les grands vases pour travailler de l'intérieur et j'en suis arrivé à faire ce grand mur où j'étais devant et derrière, et cela ça rajoute quelque chose. Travailler par devant et par derrière, ça introduit un élément très intéressant. Parce qu'il y a une espèce de rapport frontal avec le tableau. Et puis j'ai fait des tableaux parterre, au plafond, au mur. Josef m'a convaincu de faire la performance ensemble. Parce que dès que je restais à l'extérieur et que je dirigeais les danseurs ça devenait gratuit et décoratif. Quelque chose ne marchait pas. Ensuite, quand on a commencé à le faire, tout de suite j'ai vu qu'il y avait quelque chose d'intéressant. Les outils sont ceux que j'utilise pour mon travail avec l'argile. Les chaussures sont des chaussures de golf avec des crampons pour ne pas glisser. Certains outils viennent aussi d'Afrique pour creuser des épuisettes ou des choses qu'on utilise pour semer, d'autres sont fabriqués express : des massues...

7 Paul Ardenne : Josef Nadj est chorégraphe. Il se met souvent lui-même en scène donc en tant qu'acteur. A-t-il souhaité donner un sens chorégraphique à cette création, a-t-il voulu d'une certaine manière la métrer, l'écrire, lui donner un rythme particulier?

8 Miquel Barceló : On a laissé un peu les choses se faire, en travaillant et en improvisant beaucoup et puis je pense qu'on a fait un travail de nettoyage de toute narration, de tout élément théâtral. Je me rappelle au début à Avignon c'était beaucoup plus chargé et on est arrivé vraiment au travail avec la matière. Bien sûr le fait de sortir habillé en noir tout propre sur un grand mur d'argile, donne forcément un aspect théatral. Et puis le fait de le répéter des dizaines, peut-être des centaines de fois. Je crois qu'on l'on la jouée plus de 100 fois cette chose.

9 Paul Ardenne : Puisque notre sujet de réflexion est la performance, il est question aussi d'explorer le rituel et le rapport avec des pratiques qui mettent en jeu le mouvement du corps à des fins qui pourraient avoir une connexion avec le sacré. Est-ce que toi tu as eu des images, mêmes subliminales, sur tout ce qui touche dans les mythes à la terre ? Question que je pose parce que tout ton travail, depuis la fin des années '70 est vraiment imprégné par la question de la mythologie, des origines et du retour à quelque chose d'essentiel, presque de " primitif ».

10 Miquel Barceló : Il y a deux éléments qui sont très décisifs dans Paso Doble. D'abord l'origine de cette pièce est à rechercher dans cette œuvre dans la cathédrale de Palma. Il s'agit d'un sacré, où se déroule la messe. Je m'y suis introduit de manière un peu abusive, et puis ça a donné Paso Doble, qui est la version live de cette chose. L'autre source est assez évidente : il s'agit de mon expérience africaine, je vis au Mali depuis vingt ans, chez les Dogons notamment. La manière de crépir les maisons chaque année avec le banco, se fait à la main et ça s'arrondit chaque année. Ce sont des gestes très 
proches à ceux que j'utilise ici. L'argile est tapée comme de la chair. C'est une architecture très organique. Et comme il y a des pierres et du bois à l'intérieur, l'argile prend une forme. Ensuite, il y a des mottes d'argiles qui sèchent au soleil. Beaucoup de choses que nous appelons « terre cuite » finalement est de la terre crue séchée, un peu fissurée. J'aimais bien l'idée de la terre qui reste toujours molle, qui ne sèche jamais. C'est comme un traité de peinture, c'est drôle de parler de peinture avec de l'argile, mais pour moi c'est de la peinture... J'ai remarqué aussi que le son de l'argile est très proche de celui de la chaire. Comme le corps, l'argile garde la mémoire de chaque geste, qu'il s'agisse d'une caresse ou coup de poing.

11 Paul Ardenne : Ce qui m'a toujours frappé, c'est tout le travail avec les masques, le fait de couvrir le corps d'argile et l'intensité du travail, c'est extrêmement physique, fatiguant, épuisant.

Miquel Barceló : On arrive, tous les deux, à la limite. A Avignon j'avais du mal à savoir ma limite, en plus il faisait très chaud. Oui, c'est extrêmement physique, j'aime beaucoup ça, c'est très intense à chaque fois. Quand je fais des nouveaux masques et des nouvelles images, j'aime bien voir jusqu'à quel point ça peut changer. Quand je vois ça, je le reconnais à peine. C'est tellement différent déjà. Si cette œuvre peut se renouveler, c'est qu'on peut encore la jouer.

13 Paul Ardenne : Cette fatigue permet-elle de retrouver une proximité avec la transe? Cela est-il recherché ?

Miquel Barceló : Sûrement.

15 Paul Ardenne: Ce sont des gestes immémoriaux presque inscrits dans nos gênes humains. Peut-être un mot sur la violence, la violence de l'exécution?

16 Miquel Barceló : Cette violence m'a étonné, car au début c'était plus drôle. Au début on rigolait beaucoup. Et je voyais que le public rigolait aussi beaucoup. Petit à petit on s'est probablement rapproché de quelque chose qui n'est pas loin de la transe, arrivé dans quelque chose que je n'arrive plus à qualifier, mais en tous cas on rigole plus! Il y a un moment extrêmement violent et même dangereux, Nadj s'est même évanoui à deux reprises pendant quelques minutes sur scène. Une fois, je croyais que je l'avais tué ! J'étais même mal à l'aise.

Paul Ardenne : Ce qui m'a frappé dans cette composition c'est le côté ludique, le côté du jeu. Comme des enfants, les enfants adorent: jouer avec la terre. S'agit-il d'une régression jusqu'au stade anal comme peut-être demanderaient nos amis psychanalystes?

18 On voit Josef Nadj creuser un trou et puis toi qui rentres aussi dedans.

19 Miquel Barceló : Tous les deux nous creusons un trou. Ces deux disparitions créent deux trous, et ces deux trous finissent le dessin. Ce sont les deux orbites d'une tête. Voilà une grande image définitive.

20 Paul Ardenne : Je me demande si un archéologue du futur qui découvrait ce qui reste de cette œuvre dans 30.000 ans l'associerait à l'art contemporain ou finalement à des pratiques rituelles. Je ne sais pas s'il ferait la différence. Je ne sais d'ailleurs pas s'il y a une différence.

21 Miquel Barceló : Moi je ne crois pas qu'il y en ait. Je ne pense pas que ce soit vraiment très important. Moi j'y vois plein de références à l'art contemporain, au mien et à celui des autres. Quand j'ai joué à Athènes la même Paso Doble, j'allais tout le temps voir les 
céramiques cycladiques et c'était très drôle de retrouver les mêmes images que dans Paso Doble.

Agusti Torres Domenge, Paso Doble. Performance de Miquel Barceló et Josef Nadj, 2006.

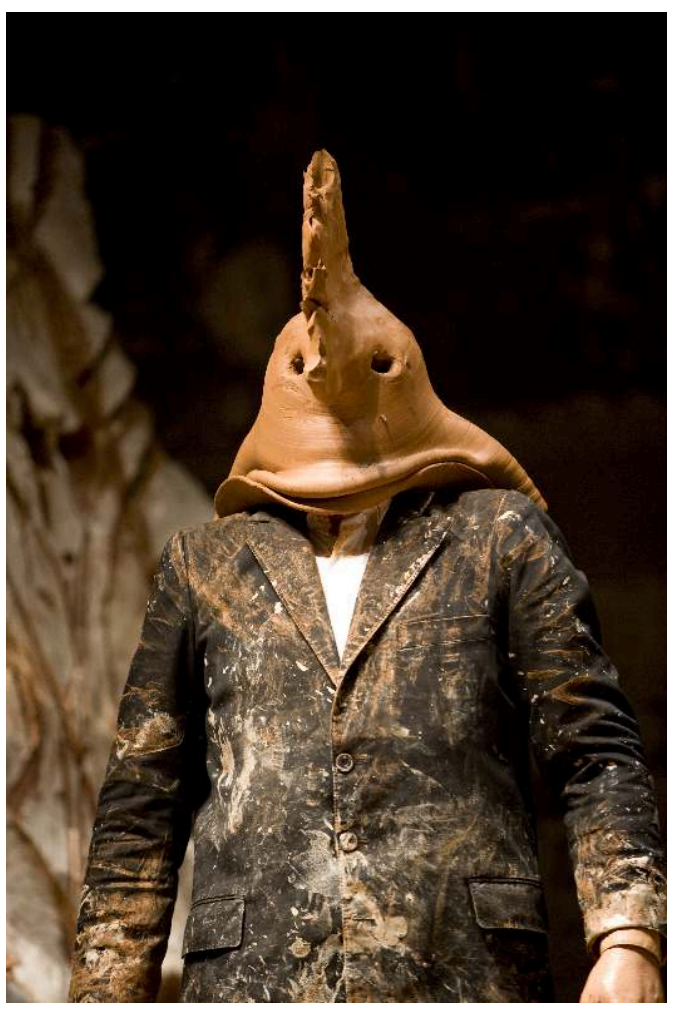

Paul Ardenne : Je vais essayer de rebondir sur le travail Paso Doble en m'interrogeant maintenant sur la performance artistique, sur le rituel et sur le jeu des connexions, à savoir d'ailleurs s'il y en a. Eu égard au thème de ma conférence, quelle obligation de recherche? Rien moins qu'établir d'éventuelles passerelles entre la performance artistique contemporaine, qui ressort du domaine de l'art, et certains rituels propres aux civilisations vivant selon un régime ante-moderne ou traditionaliste, redevables, pour leur part, du sacré.

« Paso Doble » signifie « pas redoublé » et désigne selon le dictionnaire une « danse sur une musique de caractère espagnol à mouvement rapide, à la mode entre les deux guerres ». Du titre de l'œuvre du tandem Barcelo-Nadj, on retiendra d'abord que c'est moins une danse stricto sensu qu'une "performance », un jeu d'acteurs. Il s'agit d'une représentation plutôt que d'une performance, dans la mesure où l'œuvre a été donnée à voir plusieurs fois, d'abord au cloître des Célestins, à Avignon, il y a trois ans, puis en divers lieux, comme le serait une pièce de théâtre. De Paso Doble, je retiendrai aussi, plutôt que le pas particulier qui caractérise cette danse, le caractère de ce qui est fait à deux, en double, une donnée d'office importante. Ici, ce n'est pas d'abord un état de solitude qui est exposé sur scène mais une réalisation commune - le travail de modelage, de moulage ou de traçage, pendant quarante minutes environ, d'un mur d'argile et de différents accessoires eux aussi en argile. Les deux protagonistes utilisent diversement mur d'argile et accessoires : comme s'il s'agissait d'une surface où dessiner et écrire, ou d'une pâte à modeler, ou encore d'une matière au moyen de laquelle, une fois celle-ci sculptée, on couvre et on prolonge son propre corps en lui donnant 
masques ou appendices parfois empruntés au registre animalier, ou enfin d'un refuge intime où l'on finit par s'engouffrer.

Paso Doble par Barcelo-Nadj peut être étudié en soi, en tant qu'œuvre spécifique produite par la créativité débridée des intervenants, par leur sens esthétique sûr, par leur goût de l'effort et du jeu et par la passionnante révélation de l'œuvre qu'ils mettent en scène. Il s'agit d'une œuvre montrée dans son dévoilement instantané, comme peuvent l'être les peintures sur verre de Picasso que filme Henri-Georges Clouzot dans Le Mystère Picasso, une véritable leçon en direct sur l'aléthéia, cette révélation, saisie dans son instant $\mathrm{T}$, de la vérité formelle et symbolique. On pourra aussi faire de cette réalisation un exemple passionnant d'absorbent, pour reprendre les termes de l'historien de l'art américain Michael Fried : les deux protagonistes en action, ici, s'investissent avec intensité dans la réalisation de l'œuvre, une réalisation qu'on devine programmée (elle suit un déroulement particulier, à l'évidence défini à l'avance) mais qui requiert aussi, dans son effectuation et pour être réalisée, une grande concentration, une mobilisation psychique et physique de tous les instants.

Mon propos, cependant, plutôt que se fixer sur ces points, aura soin de préciser quels rapports, étroits ou lointains, cette œuvre en vérité tardive entretient avec certaines pratiques performatives ou rituelles qui recourent comme elle à la mise en jeu d'un corps humain aux prises avec la matière molle d'une part, avec le recouvrement corporel d'autre part, avec la fusion corps-matière enfin.

Paso Doble, entre autres sollicitations référentielles, convie à réfléchir à ce premier point : le rapport à la matière molle.

27 La matière molle, c'est peut être beaucoup de choses, que les artistes n'ont eu d'ailleurs de cesse d'utiliser (je pense ici à la recension qu'a fait de cet usage du mou Maurice Fréchuret dans son livre Le Mou et ses formes) : le kapok dont se sert le sculpteur pop Claes Oldenburg, la graisse ou le feutre présents dans nombre d'œuvres de Joseph Beuys, la terre qui est la base des sculptures du Sénégalais Ousmane Sow, les excréments, la base de ceux d'artistes tels Piero Manzoni, Gérard Gasiorowski, Jacques Lizène ou encore Wim Delvoye, parmi tant d'autres (un matériau pas absent de l'œuvre de Miquel Barcelo, du moins au registre de la représentation, Barcelo dont l'une des peintures montre un homme vu de dos en train de déféquer, en passe sans doute de jouer avec cette matière fécale présentée dans ce cas comme constitutive du corps même, puisque de la même couleur que celui-ci). Autre matériau mou, l'argile bien sûr, utilisée, elle depuis toujours, de matière immémoriale, à des fins de modelage, un matériau dont est par ailleurs familier Miquel Barcelo, qui l'utilise fréquemment, y compris de manière monumentale, comme par exemple dans son grand mur réalisé dans un des édifices religieux de Majorque.

La notion de matière molle est importante pour cette première raison : il s'agit toujours peu ou prou, pour qui la manipule, d'une matière complice, qui résiste peu à sa mise en forme, qui est pour l'essentiel aisée à travailler et à transformer : la matière propice par excellence aux métamorphoses. Tous les sculpteurs qui recourent au modelage le disent. Où travailler le bois ou, plus encore, la pierre requiert une concentration de tous les instants, et beaucoup d'efforts, travailler la matière molle conduit vite à l'euphorie, libère l'imagination, décuple le désir de la forme vagabonde. Seconde caractéristique de la matière molle, mais aux effets inverses cette fois: sa viscosité et son caractère collant, gluant, son excès d'adhérence. La matière, s'agissant de l'argile, résiste cette 
fois plus que de rigueur. Si on peut lui donner la forme souhaitée sans trop de mal, reste qu'elle laisse sur vous son empreinte, un peu de sa matière même.

Deuxième aspect notoire de Paso Doble, au regard du rapport avec la matière : l'acte de recouvrement corporel dont cette performance d'acteurs est l'objet et le moment élu. Quand bien même les intervenants, ici, souhaiteraient rester propres, ils ne le peuvent pas, l'argile colle à leurs doigts, à leurs chaussures, à leurs vêtements - à leur esprit, sans doute aussi. Matière physique et matière métaphysique d'un même allant, l'argile semble parée de vertus sensuelles mais aussi intellectuelles. Avec ce matériau, les protagonistes engagent un dialogue médité, matériau dont ils se servent comme d'un médium dans toutes les acceptions de ce terme - ce au moyen de quoi l'on fixe des représentations; ce qui rend propice l'expression d'une certaine vérité mise dans les figures ou dont celles-ci seraient porteuses. Le recouvrement corporel, dans Paso Doble, est au demeurant poussé à son comble : si ce n'est avec l'argile solide elle-même, ce sera au moyen d'une lance d'incendie alimentée en argile liquide que l'un des intervenants de Paso Doble va se voir recouvert, puissant crachoir avec lequel Miquel Barcelo, dans la dernière partie du spectacle, arrose copieusement, et le mur d'argile, et Josef Nadj se trouvant devant celui-ci.

Du recouvrement corporel à la fusion corps-matière, il n'y a pas même un pas : les deux sont liés aussi étroitement qu'intimement. On peut se recouvrir de matière pour se grimer, pour modifier son apparence, pour se salir, même. Il n'en reste pas moins que la matière, en plus d'orner le corps pour le meilleur ou pour le pire, dépasse le statut d'objet cosmétique (Kosmétikos, en grec : le fard; par extension, le maquillage, l'artifice de l'apparence). La matière, sitôt qu'elle recouvre le corps, le modifie signalétiquement et substantiellement : une fois chargé de matière, le corps, de facto, n'est plus le même, au registre organique comme au registre symbolique. Se fondre dans la matière, être la matière ou deux matières à la fois - celle de soi-même, la chair, et celle, ici, de l'argile c'est, en tant qu'individu, opérer pour soi une métamorphose, incarner un statut de changement physique et ontologique, endosser la responsabilité d'être une autre figure, un autre: personnage, un être autre. Il n'est pas douteux, à cette entrée, que Paso Doble tient à la fois d'une euphorique libération de soi, qui relève du jeu, et de la plus complexe ré-confection de soi, qui relève, celle-là, de l'expression rituelle.

Les productions de l'art ont vocation à rameuter les souvenirs, ce sont des catalyseurs de mémoire. Pour cette raison élémentaire: l'autonomie artistique n'existe pas, contrairement à ce qu'ont pu rêver bien des artistes modernistes. Se confronter à Paso Doble, à cet égard, équivaut à parcourir un panorama mental transhistorique dans lequel vont défiler, comme autant de points de référence, un nombre incalculable de propositions qui s'en découvrent proches, appartenant soit à l'expression rituelle, soit à l'expression artistique, celles-ci renverraient-elles à des pratiques très anciennes dans le temps. C'est là, au regard de l'historien de l'art, un des intérêts de cette création, sa capacité au transfert. Création excitante en soi, Paso Doble l'est aussi par sa puissante capacité d'emport, comme on le dit dans le vocabulaire aéronautique à propos des charges que peut soulever tel ou tel aéronef.

C'est de cet emport - dans ce cas : la charge de mémoire recélée par Paso Doble - que je veux parler à présent, en me focalisant sur les expressions rituelles ou artistiques qui toutes convoquent la triade matériau mou, couverture corporelle et fusion avec la matière. Expressions dont je fournirai rapidement, pour commencer, quelques exemples, avant d'en analyser les connexions avec Paso Doble. 

d'Océanie. Parce que le corps exposé au danger devient l'ennemi d'une force qui veut son anéantissement, le protéger s'impose. Toutes les civilisations forment ce vœu : un corps humain qui soit assez fort pour ne faire l'objet d'aucune agression. Certaines pratiques rituelles de peuples dits "primitifs » font à leur manière propre état d'un semblable constat, et s'en guérissent par un même acte symbolique de protection, via notamment le recours à l'acte de couverture du corps. Un mythe Asoro narre ainsi cette histoire : attaquée, la tribu Asoro doit quitter ses terres et se retrancher sur les rives argileuses d'un fleuve. Envoyés pour apprécier la nouvelle situation, des éclaireurs Asoros se traînent dans la boue pour ne pas être vus, leurs corps bientôt enduits d'argile, une argile qui se transforme, une fois séchée, en armure. Croyant voir dans ces mudmen des esprits nocifs venus les persécuter, les ennemis de la tribu détalent... En conséquence de quoi chaque année, comme le veut leur calendrier rituel, les Asoros célèbrent cette victoire mythique en se recouvrant le corps de boue.

Autre rituel intéressant, la fête de la boue de Mitsubori, au Japon. Voici ce qu'écrit, à son propos, l'ethnologue Itô Hiroko, que je cite in extenso : « Étrange fête de la boue ! Les jeunes mariés de l'année, bombardés de mottes de terre, se plongent dans une mare boueuse et, par trois fois, la traversent en y traînant un palanquin sacré où l'on a fait descendre une divinité. Le Japon a-t-il la spécialité d'aussi déroutants rituels ? Il semble que non! Le polémiste bouddhiste du VIe siècle qui, parlant de la fête taoïste du "Jeûne de boue et de charbon", montre les participants "se roulant dans la boue comme des ânes, s'enduisant le visage de terre jaune et, leur peigne enlevé, laissant pendre leurs cheveux..." ${ }^{1}$ atteste l'existence de ces rites en Chine. Loin d'être un rituel de repentance, le bain de boue japonais a une tout autre signification. Se battre dans la boue, s'enduire le corps et le visage sont des actes très fréquents, lors des cérémonies du repiquage des rizières sacrées. On en avance l'explication suivante: l'énergie vivifiante et fécondante des esprits divins du riz, s'incarnait dans les repiqueuses au cours d'une nuit de retraite à la ferme. Le lendemain, ces dernières la faisaient passer dans les plants qu'elles fichaient dans la rizière inondée ; l'eau boueuse se chargeait de l'énergie fécondante divine. Y agiter un mât, s'y battre, s'y rouler, recréait les forces masculines, s'en faire un masque, assurait la grossesse. (Les "hauts sourcils" appliqués avec l'index trempé dans le fard au sommet du front sont, peut-être, le reliquat de ce rituel). À Mitsubori, le rituel de la fécondité est encore plus explicite. Jadis, on traînait dans l'étang boueux un tronc d'arbre, réceptacle de la divinité. Le tronc-réceptacle a été remplacé par un palanquin à l'époque d'Edo. Mais le sens du rite n'a pas changé : les jeunes mariés assurent la fécondation du sol et en se plongeant dans la boue chargée de l'énergie de la divinité qu'on y traîne, ils seront sûrs de procréer dans l'année. L'inévitable duel au sabre dans une mare de boue du cinéma populaire japonais, loin de manifester une propension à des goûts pervers, n'est, peut-être, que le souvenir inconscient du bain de boue rituel : les combattants y puisent un regain d'énergie $»^{2}$.

Fin dette longue mais instructive citation, qui nous renseigne sur les mobiles de la proximité sensible avec la matière visqueuse et recouvrant : la recherche de la force, la quête énergétique, la mise en contact charnelle et symbolique avec le divin tapi dans la boue.

Autre exemple emprunté au registre rituel, avant de passer au registre artistique : le rituel vaudou, en Haïti, de la source Balan, qui a lieu au moment du Noël chrétien. Je voudrais citer à son propos Françoise Gründ : «Pendant certaines périodes de l'année, 
et en particulier celle du Noël chrétien, les paysans d'Haïti se rendent en longues processions à la source Balan. L'eau sulfureuse jaillit à l'intérieur d'une grotte. Elle abriterait de nombreux loas (esprits). Ils préparent des offrandes puis entrent dans la vasque naturelle. Brusquement une jeune femme se convulse. Possédée par Damballah la couleuvre, elle émet de petits sifflements du bout de la langue. À proximité du pont rocheux, la faible profondeur de la mare accueille bientôt d'autres corps palpitants, secoués de frissons et de tremblements. L'eau se mêle à la terre qui, soulevée, piétinée, jetée, se change en boue visqueuse. Chacun se coule alors dans cet élément qui rappelle le sang utérin. La boue grise s'insinue dans la bouche, les oreilles, les narines. Elle couvre entièrement les cheveux, aveugle les possédés, qui l'avalent en hurlant. Ils deviennent eux-mêmes cette matière mi-liquide mi-solide qui les fait glisser les uns sur les autres et les unit plus solidement que les fils de la trame d'une étoffe $»^{3}$.

Ces rituels de bains boueux ou de recouvrements de matière molle, de manière parfois proche du mimétisme pur et simple, trouvent un équivalent sur la scène de l'art, à partir en particulier des années 1950, moment où se développe la performance et où le corps devient «objet d'art», sans l'intermédiaire que peut représenter la toile. J'en citerai quelques exemples tout en tentant d'accréditer la thèse d'une liaison symbolique vraisemblable entre rituels conventionnels et performances artistiques, et ce, nonobstant leur destination respective, par principe et par vocation différente, le premier se donnant comme ouvertement propitiatoire, le second, comme incarnant et démonstratif.

Parmi les performances mettant en jeu la triade matière-recouvrement corporel et fusion avec la matière, les plus connues sont légitimement celles qu'entreprennent au tournant des années 1950-1960 les actionnistes viennois et particulièrement, parmi ceux-ci, Gunter Brus et Otto Mühl. Brus, à maintes reprises, dans le cadre de ses Actions Ana (visant l'« analyse du corps ", selon le registre créatif de cet artiste) : Brus paraît sur scène ou en ville nu et couvert de peinture blanche et noire, comme s'il était devenu à la fois un objet signalétique et un être mutant. Les performances d'Otto Mühl intensifient la procédure $\mathrm{du}$ recouvrement corporel et de la métamorphose métaphorique que ce dernier est censé signifier: une officiante, par l'artiste, lors de performances publiques, est recouverte de boue, de matière fécale, ou d'immondices diverses, parfois dans une intention de réification corporelle, d'anéantissement de la corporéité (« Devenir marécage d'un corps féminin »).

Autres performances artistiques, au registre matière-recouvrement corporel et fusion avec la matière, devant attirer notre attention : celles de la Cubaine Ana Mendieta, qui va multipliant dans les années 1970, et les photographies consacrées à des vues de paysages où un élément adopte une apparence ou une attitude anthropomorphiques (les Siluetas, formes naturelles qui connotent: la forme du corps humain), et les actions physiques où elle-même se recouvre de terre et travaille à se confondre en celle-ci, jusqu'à l'anéantissement visuel.

40 Enfin - une proposition qui renvoie en droite ligne au rituel de la boue de Mitsubori évoqué à l'instant -, la performance restée fameuse de Kazuo Shiraga intitulée Lutter dans la boue : l'artiste, dans un bain de boue, essaie de se mouvoir, multipliant les efforts et, dans le même temps, cherchant un contact total avec celle-ci. Comme à vouloir s'incarner littéralement dans la figure du mudman, du corps de boue. - Volonté d'incarnation en l'occurrence pertinente au regard du mouvement artistique auquel 
appartient Shiraga, Gutai, un collectif actionniste d'avant-garde formé à Osaka autour de 1955, et dont l'intitulé signifie justement « incarnation ».

Dans tous les cas cités, qui mériteraient plus d'attention, la performance vise à asseoir, du corps même, du performer comme acteur, une position. Elle s'offre comme un processus éminent de réalisation. Enfin - l'on retrouve là une des obsessions latentes de Paso Doble mais aussi des rituels primitifs engageant un jeu tendu avec la matière molle - la performance réalise une métamorphose corporelle au terme de laquelle le corps transformé, parce qu'il est devenu autre, est de concert devenu mieux, comme rebâti, régénéré, ressuscité. Principe actif que je qualifierai, d'une manière certes peu canonique, comme celui du "Autre-mieux », du "Être autre c'est être mieux », qui fait de la performance non pas seulement une gesticulation poétique ou une chorégraphie à fins esthétiques mais une stratégie améliorative du soi, l'équivalent d'une thérapie, renvoi direct à la sotériologie, cette stratégie de la quête du salut.

Le psychanalyste Daniel Sibony remarque que "Tous les artistes sont uniques; pourtant, tous semblent avoir le même enjeu : produire le point de rencontre entre ce qu'ils aiment et ce qu'un public peut aimer; rencontre de deux narcissismes, celui de l'artiste et celui de qui regarde ; le point où ces deux amours-de-soi peuvent se mêler, se toucher $»^{4}$.

43 Aucune pratique artistique, mieux que la performance, n'avoue le narcissisme fondateur de l'art, ou, plus exactement, le "transnarcissisme» qui en régule l'économie. L'artiste agit toujours en demande de reconnaissance, et en manque d'amour. Quant au public, s'il adhère à l'offre de l'artiste, c'est à toutes fins d'être reconnu par ce dernier, à la fois valorisé et intronisé, grandi par son introduction dans la sphère hautement symbolique de l'art. L'avantage de la performance, par rapport à toute autre forme d'expression artistique, c'est le raccourci qu'elle consacre. Le transit narcissique, dans son cas, est vécu au contact, en direct, sans l'ordinaire intermédiaire de l'œuvre d'art. Nulle interposition, en effet. D'essence scénique, la performance - dite encore, selon les traditions ou les usages locaux, art "performatif », " art action », art d'« attitude" ou "manœuvre " - est une formule théâtrale, où l'artiste d'abord se «voit» (la racine étymologique, en grec, du terme "théâtre»). La confrontation directe avec le public est la garantie d'un échange concret, sans barrière, sans délai non plus, au sein d'un espace-temps fusionné de la poétique et de l'esthétique. La création et sa réception, dans un rapport instantané, y trouvant même à l'occasion cette opportunité : évoluer l'une en fonction de l'autre, muter, se corriger respectivement.

L'analogie avec l'office religieux, autant qu'avec le théâtre, est implicite. Un officiant, l'artiste, une assemblée de fidèles, le public, se sont rassemblé. Leur sujet de préoccupation, l'art, a, tout comme la religion, vocation au salut, à la médication de l'âme, à la régénération. L'officiant qu'est l'artiste, pour son public, ne réalise jamais un tour de piste stérile. L'histoire de la performance, à cet égard, nous enseigne combien celle-ci voit au contraire l'artiste s'en saisir pour se livrer le plus clair du temps à un jeu cathartique intense, le portant à ses propres limites, des limites parfois transgressées sur scène ou en tout autre lieu choisi pour l'expression (la rue, fréquemment, ou de plus en plus les grandes manifestations culturelles, en général dotées d'un volet "Performances»). Les exemples de cette qualification à dessein excessive de la performance forment pléthore, et qui en doute consultera avec fruit les inventaires précis et encyclopédiques, devenus des classiques, dressés notamment par Richard Martel ou Arnaud Labelle-Rojoux ${ }^{5}$. Philippe Stelarc, rejouant le rituel de suspension 
indien de l'O-kee-pa, se suspend nu au-dessus d'une rue new-yorkaise, des crochets de boucherie passés sous sa peau. Serge III Oldenburg, lors d'un festival parisien de la Libre expression, expérimente la roulette russe devant quatre cents personnes. Gunter Brus et Otto Muehl cités plus haut, à Vienne, brisent en public tabous et interdits liés à la sexualité et à la pudeur corporelle. Marina Abramovic, livrant son corps à l'assistance, se laisse toucher au risque de l'agression violente, etc. La performance est libératrice, désinhibitrice. On y dénoue traumatismes et défaites. On y fera valoir une capacité de résistance ou de révolte. On y démultiplie le potentiel du corps à l'épanchement, à l'arrachement. Art "corpopoétique » par excellence, dont l'artiste sinon son public attendent une rémission, un allègement de l'oppression, une suspension de la douleur, de la difficulté ou de l'étouffoir d'exister.

Le territoire physique de la performance est, avant tout autre, le corps, le corps même du performer. Toute performance est une aventure de la corporéité privée, serait-elle livrée en pâture au public. On pourra parler, la concernant, d'«extimité", par opposition à l'« intimité ». Ce qui d'ordinaire demeure privé est, là, donné à voir, livré à la connaissance d'autrui, révélé comme on révélerait un secret de famille. Ce coming out de la corporéité intime définit et délimite la singulière "géographie» de la performance. Celle-ci, sans doute, se signale par le resserrement: le performer se concentre et se recentre sur son corps, premier lieu d'expérience de toute vie propre et de toute socialité. Tout autant, cette géographie se signalera par l'expansion : le corps du performer se répand, il occupe l'espace public au nom de l'espace privé, il s'arroge le droit de se constituer comme territoire corporel envahissant, indu mais légitime.

Cette manière aiguë de faire valoir le corps en dit long sur les limites mises dans les faits à l'expression libre, l'environnement du performer serait-il celui de la démocratie, lieu politique par excellence de la liberté d'expression, du moins présumée. Non démentie depuis les premières performances futuristes du début du XXe siècle, la profusion de l'«extimité» dont fait état l'extraordinaire fécondité de la " performance » décline en fait, moins paradoxalement que logiquement, la frustration, plus que l'accomplissement. Un corps accompli n'a plus besoin de la performance, pas plus d'ailleurs qu'il n'a pas besoin de l'art. La richesse de la performance comme genre, à cet égard, est indissociable de la perpétuation de la conscience malheureuse et d'un sentiment décidément durable, celui du narcissisme non comblé. Le monde, le réel, la liberté qui nous est consentie par les appareils d'État ne sont-ils pas à notre convenance ? Heureusement, il reste la performance, qui libère et nous allège, au moins symboliquement.

Ce qui ne manque pas de frapper l'historien de l'art, concernant la performance, c'est tout à la fois la rapidité de sa constitution comme genre, sa permanence (sa cote n'a jamais faibli, même une fois passées les grandes heures des années 1950 et 1960), la nature même de sa pratique, à la fois répétitive et peu innovante. Au risque d'être taxé de mauvais esprit (mais telle n'est pas mon intention), l'on se doit de dire à cet égard combien Paso Doble est une œuvre moins novatrice que "réitératrice», qui continue l'histoire sans la bousculer plus que ça - une réalisation qui n'innove en rien, ni par ses modalités d'exécution, ni par l'esthétique qui en émane, au regard notamment de l'histoire de la performance et de ses réalisations antérieures au début du XXIe siècle. Le caractère finalement conventionnel, consensuel de cette œuvre - le fait qu'on l'apprécie immédiatement - résultent de deux données : Paso Doble, d'une part, s'inscrit dans le paysage de la création humaine comme une formule héritée, aux mobiles et au 
contenu aujourd'hui familiers; Paso Doble, d'autre part, réitère ontologiquement la pratique ritualisée, le jeu d'acteurs qui nous y est présenté réplique en droite ligne celui des officiants ayant en charge la mise bord à bord de l'humanité et du sacré tels que chamanes et autres prêtres ou sorciers, du bouddhisme au vaudou en passant par le christianisme et ses rituels d'exorcisme.

Dans un compte-rendu récent consacré à un festival de performances asiatiques dont il a été le témoin, le performer et historien de l'art Alain-Martin Richard s'étonne ${ }^{6}$. Il avoue s'être attendu à des spécificités locales, de même qu'on ne peindrait pas par exemple de la même façon à New York et à Bangkok, pour des raisons de culture vernaculaire, d'usage, de demande, etc. Mais non. En tous points, les performances réalisées en Asie auxquelles assiste Alain-Martin Richard se conforment à un modèle d'expression qui est depuis le début celui de la performance, où qu'elle se donne cours. Même théâtralisation outrée, même intensité psychologique du jeu, même volonté démonstrative, même exhibitionnisme scénique. Jusqu'aux thèmes abordés, tournant pour l'essentiel autour de la question de la position du corps - position physique, sociale, culturelle, mentale -, faisant état de correspondances évidentes.

Ces critères de continuité et d'invariance, sans trop tirer sur la corde de l'analogie, autorisent à établir un parallèle entre performance et rituel. Le rituel, dans la pratique religieuse, c'est cet acte à vocation sotériologique que l'on répète ad inifinitum, et auquel on ne saurait rien changer : le rituel, en effet, émane du dogme religieux, ou d'un usage sacralisé du geste. Tout ce que la performance vient ritualiser de la vie, sans doute, n'est pas uniforme : ni d'une performance à l'autre, ni d'un performer au regard d'un autre. Cette donnée de différenciation, ceci posé, n'enlève rien au caractère rituel de la performance, celle-ci serait-elle le lieu d'expression d'une infinité de rituels. C'est là le signe, en effet, de l'existence d'une nouvelle religion, celle du corps privé (le credo individualiste du " Moi d'abord»). De cette nouvelle religion sans autre dieu que le moi se dégagent naturellement de nouvelles pratiques crypto-religieuses, pratiques en l'occurrence propres à l'ère de la désacralisation inaugurée à large échelle par la modernité : pour l'occasion, des mises en scène du moi venant qualifier les rituels du corps d'aujourd'hui, un corps, on le sait, tout à la fois en charge de se constituer comme figure, comme histoire et comme destin. - Figure, histoire et destin, très exactement ce que nous offraient les anciennes religions, au temps de leur éminence symbolique.

Réponse individualisée à la massification (pouvoir dire "J'existe » et le montrer), la performance est tout autant l'art par excellence de l'ère individualiste désacralisée, à présent que chacun d'entre nous est devenu Dieu et peut revendiquer de l'être.

\section{Discussion}

51 Bernard Müller : Miquel Barceló a parlé du fait que sa performance a été jouée au moins une centaine de fois, et je voulais savoir s'il existait un canevas, un script ou une sorte de partition permettant tout au moins de reproduire plus ou moins à l'identique la durée de la performance. Deuxièmement, et cette question s'enchaîne à la précédente, je voudrais savoir si vous admettez une intention rituelle ou sotériologique, dans ce travail.

52 Miquel Barceló : On n’a jamais écrit une partition ; mais elle a été dite. Ensuite, dans ma pratique de peintre, je ne mesure pas vraiment le temps alors j'ai été étonné de voir que la performance durait toujours entre 45 minutes et une heure. Il existe une sorte de 
bande composée à partir des bruits que nous faisons avec la terre. Ensuite, je pense qu'il existe une origine ecclésiastique de Paso Doble, ça vient des parois d'une église dans laquelle des messes sont dites. Alors je pense qu'il y a sans doute quelque chose de ces rituels. Comme c'est une œuvre-métamorphose dès qu'on la répète plus d'une cinquantaine de fois, ça devient quelque chose d'autre. Je suis curieux de voir où ça m'amène et ce que ça devient. nouvelles religions, comme de nouvelles civilisations apparaissent, des anciennes disparaissent, donc des rituels aussi apparaissent et disparaissent. Moi je ne suis pas un spécialiste de l'histoire des religions et encore moins de l'histoire des rituels. Ce que j'ai crû comprendre, très humblement, c'est qu'il n'y a pas de pratique symbolique qui ne soit pas accompagné de rituel, et il n'est pas de rituel qui n'ait une signification très précise. Si on ne fait pas varier la signification du geste rituel, de fait, on ne fait pas varier le rituel non plus. Si vous allez assister à n'importe quelle cérémonie - si possible si vous appartenez à la religion en rapport avec cette cérémonie - il est intéressant d'imaginer que parfois des centaines, des milliers d'années avant vous, le rituel était très exactement énoncé de la même manière.

Cela vous inscrit dans une espèce de très longue durée qui excède la vie humaine et qui est la durée métaphysique. Cela renvoie à ce que Platon appelle la "chaîne aimantée ", c'est-à-dire le fait qu'il y a une espèce de lien aux dieux - entre les dieux et les hommes - et le rituel est très précisément ce qui pérennise et éternise ce lien. Alors à propos des rituels, oui, ça peut changer, même si parfois il faut en inventer. J'avais été frappé, je me souviens c'était aux USA en parlant avec quelqu'un qui cherchait à définir des rituels nouveaux dans un cas particulier : comment honorer la mémoire des gens morts du SIDA, c'est-à-dire quelle cérémonie faire. C'était exactement la question que cette personne se posait, je me rappelle en avoir parlé avec elle, c'était: "quel rituel inventer?", c'est-à-dire comment inventer des rituels qui permettent à la fois de célébrer les gens qui ont vécus, qui permettent de parler de cette maladie, qui permettent d'une certaine manière de la rejouer de manière cathartique, de rejouer la souffrance de façon cathartique, en s'en purgeant, et qui finalement fasse la preuve qu'il y a un lien entre les vivants et les morts? Je ne suis pas un spécialiste mais ça m'avait intéressé par ce que - voilà - c'étaient des gens qui cherchaient à fixer un rituel. Et rien ne dit que quand ils l'eurent fixé ils n'ont pas pensé qu'il n'était pas tout à fait adéquat, qu'il fallait le modifier, qu'il n'atteignait pas sont but. Mais je pense d'une certaine manière que finalement l'eucharistie chez les chrétiens, chez les catholiques, c'est-à-dire le moment de la communion reste le moment de la communion depuis que les règles ont été fixées par la patristique il y a quinze siècles de ça. 
Public: Je m'intéresse surtout à l'œuvre de Barcelo. Chaque fois que vous faites une performance est-ce que vous la filmez? Ce qui m'avait semblé intéressant dans le déroulement, c'est le fait qu'à chaque fois vous avez l'impression d'avoir un tableau en mouvement.

Miquel Barceló : Non, on ne filme pas. Je n'y ai pas vraiment pensé à ça. Après, je me suis dit « ah oui, on aurait du filmer ", mais on ne l'a pas fait. On a pris quelques photos, mais pas beaucoup.

Public : C'est ce qui serait original pour notre époque; c'est-à-dire qu'au début quand vous vous êtes arrêté un petit moment pour nous montrer le tableau enfoncé, je me disais : que c'était magnifique et que vous alliez tout abîmer. En revanche, vous l'avez tout simplement changé et il est beau de nouveau. C'est-à-dire que c'est une suite de tableaux : chaque performance est un musée en soi.

Miquel Barceló : Vous savez c'est un peu ce qui se passe dans l'atelier : on finit par se dire : «c'était super et j'ai tout abîmé ». C'est une phrase que j'entends souvent.

Public : Ce n'est pas « abîmé », mais il est devenu « autre »!

Miquel Barceló : Ça se métamorphose jusqu'à la disparition. C'est un cycle à chaque fois. On répète ce cycle. Alors quelque fois on prend des photos, des fois pas.

Public: Ce ne serait pas dans la perspective de documentation mais plutôt dans celle d'une esthétique qui change?

Miquel Barceló : Ça se passe devant quelques centaines de personnes et puis c'est fini.

Public : S'agirait-il d'un sort de cirque alors?

Miquel Barceló : Oui, comme le cirque ou comme la tauromachie.

Public : Je voudrais vous parler d'un événement qui va se produire et qui à mon avis à un lien avec ce dont on parle. Il s'agit d'une exposition qui va commencer en mai au Musée des Abattoirs à Toulouse qui s'appelle «Dream Time ». Elle met en place une proposition avec des artistes contemporains en relation avec la préhistoire. Une exposition qui aura lieu de mai à septembre, il me semble, aux Abattoirs et dans la grotte du Mas d'Aziil en Ariège. Les propositions tourneront autour de ce que le musée des abattoirs appelle la «transhistoire » et je voudrais savoir, comme je sais de source à peu près sûr que vous faites partie de ce projet, est-ce que vous travaillez en ce moment sur les grottes d'Ariège ou du Lot, il me semble que oui, et quelle proposition allez-vous faire?

Miquel Barceló : J'ai visité cette grotte. J'en ai visité beaucoup. Mais ma proposition n'est pas encore très définie. C'est encore en cours.

Public: Paul Ardenne, vous disiez toute à l'heure que pour résister à la massification chaque artiste incarnait un dieu et que ça rend d'autant plus particulier ce travail avec Miquel Barceló et avec Josef Nadj, par ce que là ils sont deux.

Paul Ardenne : Oui, il n'y a rien de contradictoire. Je ferai juste état d'un constat: beaucoup d'artistes travaillent en double. Le vingtième siècle a crû inventer le collectif en art; mais en réalité, j'ai envie de dire : il n'a rien inventé. Si on se reporte à ce qu'étaient les ateliers d'artistes à l'âge classique, il y avait effectivement un grand maître qui était là, qui signait, qui donnait le blanc-seing à chaque œuvre produite. Mais dans les faits les œuvres étaient faites collectivement. Quand on lit les fameuses études de Svetlana Alper sur les ateliers de Rembrandt, d'où sont sorti environ six cents toiles, on remarque que sur certaines toiles, Rembrandt n'a strictement rien fait. Il s'est 
contenté de signer. Alors, beaucoup d'artistes travaillent en double, ce qui se fait à deux ne me paraît pas venir contester la question centrale de ce qu'est la mise en forme de l'art, de ce que Bachelard appelait la « solitude organisatrice ». Mais le fait d'appuyer sa solitude sur celui d'un autre et de profiter de cette solitude ne peut que constituer une relance et une fortification, même si parfois ça peut se transformer en échec.

On est fondamentalement des êtres sociaux et on passe notre vie à faire quelque chose qui est extraordinairement difficile à réaliser et qui consiste à affirmer notre toute puissance tout en continuant à vivre avec les autres. C'est d'une violence inouie, mais ça s'appelle la vie collective.

73 Miquel Barceló : Je n'ai pas fait souvent des œuvres collectives. Seulement quand j'avais 17 ans, je faisais partie d'un groupe d'action artistique et on faisait des œuvres collectives très poétiques. Ensuite, mon travail était plutôt solitaire. Une solitude organisatrice mais aussi désorganisatrice. Et puis avec Nadj, c'était une expérience très intéressante de ce côté-là. Maintenant j'ai quelques autres projets en tandem avec un autre artiste. Avec Nadj c'était intéressant par ce qu'il venait d'une discipline différente à laquelle je ne m'intéressais pas énormément. La danse contemporaine, je connais un peu, mais je ne suis pas un expert, vraiment pas. Dans ce contexte, me forcer à monter sur scène, ce n'était vraiment pas évident, c'était loin de moi.

Public : Je voulais juste revenir sur la question du rituel parce que Miquel Barcelo en parle assez peu de son rapport au religieux entre guillemets, mais je voulais quand même essayer d'insister un peu: de savoir dans quel état êtes-vous quand vous êtes dans cette performance? Paul Ardenne vous a parlé d'un état de transe. Je remarque aussi que vous parlez peu de votre rapport au Mali où vous êtes proche des Dogons qui vous influencent certainement du point de vue spirituel. On a l'impression que vous évacuez cette dimension?

75 Miquel Barceló : Sans doute, mais Paul a parlé d'un état de transe et je crois qu'on arrive à un état de transe, sans doute. Mais j'ai un peu de mal à en parler, vous savez c'est peut-être parce que je suis toujours en train de faire Paso Doble. On va le faire à Venise bientôt, à Barcelone dans un mois. Alors je pourrais peut-être en parler un peu mieux quand ce sera fini. Dans un an ce sera la dernière représentation. Quoi qu'à Avignon on avait dit que je le ferais qu'une fois à Avignon, une fois et plus jamais, c'était ma condition sine qua non. Et voilà vous voyez, je ne suis pas à une contradiction près. Mais pour en revenir au Mali, je suis conscient qu'une grosse partie de mon inspiration vient de mon expérience africaine. C'est une œuvre encore en train de se faire. Et je laisse donc pour plus tard la réflexion théorique.

Paul Ardenne : Je voudrais juste ajouter quelque chose. Il y avait Jean de Loisy tout à l'heure. Je ne sais pas si Jean est toujours là. C'était le commissaire de cette exposition remarquable, «Traces du sacré » au centre George Pompidou. «Traces du sacré » qui a eu lieu l'an passé et que certains d'entre vous ont peut-être vus, est une exposition tout à fait intéressante, passionnante à propos de laquelle je formulerais juste une réserve, mais tout à fait amicalement, avec un respect qui n'est pas que diplomatique. J'ai envie de dire: le sacré il n'est pas toujours là où on l'attend, il n'est pas toujours très précisément là où on pourrait croire qu'il est et notamment, je pense à Andy Warhol par exemple. Nous allons avoir une exposition Andy Warhol au Grand Palais bientôt. Bon, pour moi, Andy Warhol c'est par excellence l'artiste du sacré, au XXe siècle, ce n'est pas pour rien qu'il était catholique pratiquant. Quand vous regardez toute son œuvre, qu'est-ce que c'est? C'est un travail sur la résurrection. De manière consciente 
ou inconsciente, cela n'a aucune importance. On peut toujours réduire Andy Warhol à la figure de l'artiste Pop ; à celui qui trouve des choses totalement ordinaires et qui évidemment s'en empare et en fait des œuvres d'art. Mais j'ai envie de dire qu'à travers ce travail de sublimation qu'il y a chez lui et qui consiste à récupérer les boîtes de soupe Campbell, les images de la Une de journaux même quand elles parlent de catastrophe, des vues de la chaise électrique, d'accidents de la circulation, d'émeutes raciales, en leur donnant la surcharge symbolique du travail de l'art. Ce faisant, il ressuscite ni plus ni moins ces choses là. Ce qui revient à dire qu'au fond rien n'est perdu. Rien de ce qui a été crée est perdu. Tout à droit à la résurrection. Le travail de Warhol finalement, c'est le travail de la résurrection. Et j'ai envie de dire que le plus grand artiste du sacré au XXe siècle c'est Andy Warhol. C'est au fond très compliqué de savoir où se niche le sacré.

On pourrait dire d'une certaine manière que Miquel Barceló, en vertu de son origine espagnole, est influencé par la culture chrétienne catholique. Et la culture catholique est une culture de l'amour de l'image, mais d'une image qui doit être éprouvée, dont il faut faire l'épreuve. Qu'est ce que c'est ce mur d'argile? C'est quelque chose que l'on crée, une image que l'on fait et que l'on détruit et finalement dans laquelle on vient se calfeutrer, parce que cette image est le triomphe de dieu. On pourrait presque dire les choses comme ça. C'est là que l'art devient quelque chose d'extrêmement difficile par ce qu'on peut se dire qu'à ce compte là tout l'art est une entreprise de résurrection et qu'il n'est jamais qu'une des formes dérivées de la pratique religieuse. Ce que d'ailleurs Hegel avait dit en son temps, en mettant simplement cette nuance, en disant : le temps du romantisme qui viendra après le temps les temps originaux de l'humanité, les temps primordiaux, primitifs, sera le temps de l'art, c'est-à-dire le moment où l'homme se saisit de la nature en étant incapable de faire autre chose que de l'esthétiser. Puis viendra le temps de raison où l'homme se saisira de la nature, mais là pour la comprendre; pour la dominer. Moi je crois que si l'art hégélien à quelque chose de faux, c'est que l'art constitue sans doute le stade suprême de la rationalité, mais à sa manière à lui, c'est-à-dire par le simulacre. Ce qui est aussi la stratégie du religieux. Le religieux apparaît toujours par le simulacre.

\section{NOTES}

1. Maspéro, Le Taoïsme, p. 418.

2. Itô Hiroko, Le rituel de boue de Mitsubori, site numérique dédié.

3. Françoise Gründ, Le Corps et le Sacré, Paris, éditions du Chêne, 2003, p. 92.

4. Daniel Sibony, Création. Essai sur l'art contemporain, Paris, Seuil, 2005, p. 5.

5. Arnaud Labelle-Rojoux, L'Acte pour l'art (édition revue et augmentée), Paris: Al Dante, 2004 ; Richard Martel, dir., Art Action 1958-1998, Québec : éditions Intervention, 2001.

6. Alain-Martin Richard, "Définir la performance asiatique », in Performances asiatiques - From Asia, publication Inter-Le Lieu, Québec, 1998, non paginé. 


\section{RÉSUMÉS}

A l'occasion de la $60^{\circ}$ édition du Festival d'Avignon en 2006, le plasticien Miquel Barceló crée avec Josef Nadj, coréographe, Paso Doble, un spectacle qui conjugue l'univers artistique de l'un et l'univers chorégraphique de l'autre. Performance à quatre mains Paso Doble met en scène Miquel Barceló et Josef Nadj, aux prises avec un mur d'argile. Quarante minutes durant, les deux protagonistes de cet exercice de sculpture en live décorent la matière molle, la triturent, s'y fondent enfin. Fruit de l'imaginaire de mudmen hors norme, cet étonnant ballet tient à la fois de l'art, du rituel, de la transe païenne ou sacrale, de la gymnastique, au choix. Paul Ardenne examine cette synthèse créative à la lumière des pratiques corporelles, artistiques ou non, élisant avec la matière molle un rapport d'élection, entre décoration primitive du corps, recouvrements rituels et performances plus récentes recourant à la terre, telle Lutter dans la boue de Kazuo Shiraga, artiste affilié au mouvement Gutai (années 1950, Japon).

In 2006, for the 60th edition of the Festival of Avignon, the painter and sculptor Miquel Barceló collaborated with the choreographer Josef Nadj, creating Paso Doble, a theatre piece combining his artistic universe with the other's choreographic world. Paso Doble brings together two artists in the creative process as they reshape a wall of wet clay. For forty minutes, the two protagonists create a sculpture before our eyes, modelling and manipulating the soft clay until they become one with it. Fruit of the imagination of these two exceptional mud men, this surprising ballet relates to art, ritual, pagan and sacral trance, and even gymnastics. Paul Ardenne examines this creative collaboration in light of how artists and non-artists work with the body. He also explores the soft matter of clay as it evokes primitive body decoration, ritual coverings, and performance art that uses earth as its principal medium, such as the now-famous Challenge to the Mud by Kazuo Shiraga, an artist affiliated with the Gutai movement in 1950s Japan.

\section{AUTEURS}

\section{PAUL ARDENNE}

Ecrivain, historien de l'art. Universitaire (Faculté des Arts, Amiens), collaborateur, entre autres, des revues Art press et Archistorm, Paul Ardenne est l'auteur de plusieurs ouvrages ayant trait à l'esthétique actuelle : Art, l'âge contemporain (1997), L'Art dans son moment politique (2000), L'Image Corps (2001), Un Art contextuel (2002), Portraiturés (2003), outre diverses monographies d'architectes, un essai sur l'urbanité contemporaine, Terre habitée (2005), et deux romans. Autres publications : Extrême - Esthétiques de la limite dépassée (2006), Images-Monde. De l'événement au documentaire (avec Régis Durand, 2007), Working Men. Art contemporain et travail (avec Barbara Polla, 2008), À Flux tendu. La création plastique au tournant du 21ème siècle (2009).

\section{MIQUEL BARCELÓ}

Artiste espagnol, Miquel Barceló est l'un des artistes contemporains les plus en vue sur la scène artistique internationale. Ses travaux sont orientés vers le dessin, la peinture, mais aussi la sculpture, la céramique et la performance comme supports de création artistique alternatifs. L'artiste vient entre autres de terminer une intervention à l'ONU (Palais des Nations Unis à Genève) sur l'immense voûte de la « Salle des droits de l'homme et de l'alliance des 
civilisations ", un espace circulaire de 1500 mètres carrés. Depuis quelques années, Miquel Barceló vit et travaille en alternance à Majorque, à Paris et au Mali. 